\title{
Characterizing maser polarization: effects of saturation, anisotropic pumping, and hyperfine structure (Corrigendum)
}

\author{
Boy Lankhaar and Wouter Vlemmings
}

\begin{abstract}
Department of Space, Earth and Environment, Chalmers University of Technology, Onsala Space Observatory, 43992 Onsala, Sweden e-mail: boy.lankhaar@chalmers.se
\end{abstract}

A\&A 628, A14 (2019) https://doi .org/10.1051/0004-6361/201935064

Key words. methods: numerical - masers - polarization - stars: magnetic fields - errata, addenda

The polarization angles that are reported in Lankhaar \& Vlemmings (2019) have to be revisited. It has been pointed out to us that a bug in our code lead to the wrong sign of the Stokes parameters

$Q(\omega) \rightarrow-Q(\omega)$

$U(\omega) \rightarrow-U(\omega)$.

This error has no influence on the total maser brightness, the total polarization fraction, or the circular polarization, but it leads to an erroneous polarization angle. The polarization angle that we report in Lankhaar \& Vlemmings (2019), $\chi$, is related to the correct polarization angle, $\chi^{\prime}$, by

$\chi \rightarrow \chi^{\prime}= \begin{cases}\chi-\pi / 2 & \text { for } \chi \geq 0 \\ \chi+\pi / 2 & \text { for } \chi<0 .\end{cases}$

The error has lead to erroneous statements in our discussion in the paragraph beginning with " $90^{\circ}$ flip of the polarization angle." We state that when $g \Omega \gtrsim 100 R$ and propagation angles $\theta<\theta_{m}$, the polarization is oriented perpendicular to the projected magnetic field direction and for $\theta>\theta_{m}$ the polarization is oriented parallel to the projected magnetic field. Using Eq. (1), this statement should be the other way around: When $g \Omega \gtrsim 100 R$ and propagation angles $\theta>\theta_{m}$, the polarization is oriented perpendicular to the projected magnetic field direction and for $\theta<\theta_{m}$ the polarization is oriented parallel to the projected magnetic field. The polarization flip we discuss is still present. We also state in this paragraph that in the case $R \gg g \Omega$, the polarization is oriented parallel to the projected magnetic field direction. This should be corrected according to Eq. (1): For $R \gg g \Omega$, the polarization is oriented perpendicular to the projected magnetic field direction.

In Figures (1c), (2b), (3), (4b,d), (7b), (8b), and (9), the polarization angles that we plotted have to be transformed according to Eq. (1). The same goes for the appended figures (A1b,e), (A2b,e,h), (A3b,e,h), (A4b,e), (A5b,e,h), (A6b,e,h), (A7b,e), (A8b,e,h), (A9b,e,h), (A10b,e), (A11b,e,h), (A12b,e), (A13b,e), (A14b,e), (A15b,e), (A16b,e), (A17b,e), (A18b,e), (A19b,e,h), (A20b,e,h), (A21b,e,h), and (A22b,e,h).

Acknowledgements. We thank Gabriele Surcis for pointing out the wrongly predicted polarization angles to us.

\section{References}

Lankhaar, B., \& Vlemmings, W. 2019, A\&A, 628, A14 